\title{
Governmental Regulatory Issues-Reducing Uncertainties in Food Safety
}

\author{
Richard J. Ronk, James H. Maryanksi, and Patricia Thompson \\ Center for Food Safety and Applied Nutrition, Food and Drug Administration, \\ 200 C Street, S.W., Washington, DC 20204
}

Introduction

Eve: "Here is an apple for You."

Adam: "Is it GRAS?"

Eve: "The serpent said it was safe" (Reitz and Caldwell, 1974)

The safety of our food supply has been an issue recognized in public policy, government, legislation. and regulation for many years. A basic tenant of food safety is that "food should be safe, sound, wholesome and fit for human consumption" (Biotechnology in Food Processing). Technological innovations in recent years have greatly enhanced our ability to ensure an abundant supply of food that can be distributed over great distances and stored and pro cessed for our convenience. At the same time, scientific advances allow the chemist to detect minute quantities of impurities in food and the molecular biologist to manipulate the very fabric of the genome. Accompanying these advances are many complex scientific questions to be answered to ensure safety for human health and for the environment. While new advances have occurred. such as the advent of modem biotechnology, many of the questions that are being raised have been with us a long time. In a sense, we are deal ing with uncertainty, and through scientific investigation we expect to achieve a level of comfort that will reduce that initial uncertainty to a reasonable certainty that no harm will occur before new products can be accepted for the market. The Food and Drug Administration (FDA) has taken actions to ensure that uncertainties in the food supply are reduced. For example, the agency published a proposal in 1970 that indicated that the federal government would review new cultivars of food crops that has been modified significantly by conventional plant breeding (Federal Register, 1970). At that time, consideration was also given to specifically reviewing new varieties that exhibited either a) a $10 \%$ increase in a native toxicant, or b) a $20 \%$ decrease in an important nutrient. This proposal raised considerable response from the industry, and the agricultural community in general, because of the arbitrary nature of the propos al and because of fears of increased government oversight. Nevertheless, discussions of the proposal generated an increased awareness of the importance of monitoring various problems that could arise, and current agency regulations state that FDA can review new cultivars of crops that have been significantly altered by breeding and selection (21CFR170.30).

Modem biotechnology has expanded greatly the possibilities for developing improved crops through techniques that permit more rapid and precise modification of genetic traits. Essentially, any gene that can be identified can be transferred to any recipient organism without respect for natural barriers to gene transfer. The first examples of the use of modem biotechnology in agriculture have been to develop plants that resist pests and diseases. that are tolerant to herbicides, that exhibit improved fruit ripening, or that have improved nutritional profiles. How should the safety of these products be examined? How should they be regulated? What contribution can agricultural science, including modem biotechnology. make toward a safer food supply? These issues will be discussed, but first, FDA's role and authority in protecting the food supply will be described.

\section{FDA's authority}

The agency's authority under the Federal Food, Drug. and Cosmetic Act over food and food ingredients centers on the product that is introduced into interstate. commerce, rather than the method by which it was produced. FDA authority extends from the farm to the consumer. Because it is the agency's primary responsibility to ensure a safe and wholesome food supply for the nation, FDA views biotechnology in the broadest context. Regardless of the methods applied (chemical, physical. or biological) to produce new food and food ingredients, the safety of new products must be assessed under the act.

Several years ago, FDA carefully considered how food products of biotechnology should be regulated, and the Agency concluded that new laws are not needed to ensure the safety of food products developed through recombinant DNA techniques or other applications of biotechnology. Although there are no statutory provisions or regulations that address new technologies. FDA possesses extensive experience in the safety evaluation of products made by tradetional methods, and food products derived by new technologies can be adequately regulated within the framework of the existing law. These and other general policies of FDA concerning the application of new technology to food products were published in 1986 under the Office of Science and Technology Policy's "Coordinated Framework for Biotechnology” (Federal Register, 1986).

\section{Legal statutes and regulations}

A discussion of genetically modified agricultural crops and the agency's role in assessing their safety must involve a consideration of the pertinent laws and regulations. Most issues concerning the safety of a food involve the application of the food adulteration Provisions (section 402). the food additive provisions (section 409), or the color additive provisions (section 706) of the Act. The food 
adulteration provisions provide, in part. that a food is adulterated if it bears or contains any poisonous or deleterious "added substance" that may render it injurious to health. Courts have agreed with the agency's interpretation of this section that any substance that is not an inherent constituent of food may be regulated as an "added sub stance." Furthermore. if the quantity of a food constituent exceeds the amount that would normally be present because of some techno logical adjustment to the product, the excess quantity may also be viewed as an "added substance".

The other statutory provisions of the Act used by the agency in determining the safety of food and food ingredients are the food ad ditive provisions [section 201(s) and 409] and the color additive provisions [sections 201(t) and 706]. The Act required that substances employed to color food be used as prescribed by regulation. and, therefore, requires premarket approval for color additives. The definition of a food additive [section 201(s) of the Act] includes both artificial and natural substances and provides, in part, that the term "food additive" means any substance that, through its intended use, may become a component or affect the characteristics of any food. unless the substance is generally recognized as safe (GRAS) by qualified experts. Premarket approval is required for food addi tives. and industry frequently requests the agency's opinion concerning GRAS status of a food product.

The agency's procedural regulations for GRAS food ingredients are described in the Code of Federal Regulations (21 CFR 170.30) and state that the agency will review the GRAS status of ingredients of natural biological origin that have been widely used for nutrient properties and that have been significantly altered by breeding and selection or by the manufacturing process. The agency intends to review new methods of manufacture for food products by the same criteria used for products derived by traditional means. It is impor tant to evaluate whether new methods result in changes in the chemical identity of the product. the introduction into the food sup ply of new or altered levels of impurities, or an increase in dietary exposure of consumers to the product that is not justified by avail able safety data. If the agency finds that the food products manufactured by a new method has been altered significantly, the FDA will either conclude that the new product also is GRAS, or that it is not GRAS, and therefore requires premarket approval under the food additive provisions of section 409 of the Act.

Most traditional staple food is considered to be GRAS on the basis of a history of safe use prior to 1958 . The central question to be considered when a food (e.g., tomato, carrot, wheat. beef, or eggs) is modified by chemical, physical, or biological processes is: "Is the modified food or food ingredient still GRAS?" The question must focus on the commercial product. not the process by which the product was derived.

Evaluating the safety and regulatory status of a new or modified food or food ingredient is a matter of considering the nature of the product in relation to its "relatives" that are already a part of our food supply. FDA would not use the same criteria to evaluate a new tomato or cucumber that would apply to other products regulat ed by the agency, such as a new human drug or a new medical device; rather, FDA needs to determine whether that vegetable is sufficiently similar to existing products within the known "template" for vegetables. Similarly, the agency evaluates potential risks of new food or color additives or GRAS food ingredients in relation to existing substances. If a new product does nor raise an increased risk when compared to its template relatives, that it should pass the test.

The development of agricultural crops through modem biotech nology will raise challenging issues for industry and for FDA. Some examples of the questions with which the agency will be faced are: How should the product of a gene that has been introduced into a food crop by new molecular techniques be viewed in a regulatory sense? What would be the status of a tomato that contains the polygalacturonase antisense gene to control fruit ripening? Or, the tomato or cucumber that has \&en modified to resist viral disease by incorporation of a piece of the virus coat protein'? The Act does not provide premarket approval for food, and FDA has reviewed only occasionally new types of food crops, such as canola.
The Food Additives Amendment of 1958 seems to dictate that any substance that is intended to become a component of food may be a food additive, unless the substance can be shown to be GRAS. Does this mean that a substance that is added to a food crop by genetic modification is a food additive by definition? Certainly. that substance has been introduced through human intervention because gene manipulations are very resource-intensive. However, conventional plant breeding since 1958 also has resulted in new traits. and thus new substances added to food. Is FDA to distinguish the new from the conventional techniques on the basis that nature is essentially accomplishing traditional manipulations and that new techniques are somehow different? It can be argued that the U.S. Congress did not contemplate that food should be subjected to the food additive definition and that the adulteration provisions of sec tion 402(a)(1) are adequate to protect the food. The definitions of food and food additive are not mutually exclusive, and this issue is not easily resolved. Additionally, new techniques provide the ability to introduce exotic traits into food crops that could not occur through conventional means and that have not been part of our food supply. It is at least possible in some circumstances that a substance that would be considered to be a food additive if it were added during food processing could now be added directly to the food crop through genetic modification. Should such a substance be precluded from the food additive definition?

These and other questions will challenge the agency and the industry to find solutions that will ensure the safety of the food supply, but will not stifle innovation of new technologies. The Act provides FDA broad authority to accomplish its mission, and the agency also has considerable discretion to decide these and other is sues.

Regardless of how these regulatory issues are settled, FDA will want to evaluate information related to the first examples of the use of modem biotechnology to ensure that the appropriate scientific questions have been answered. FDA's primary mission is to protect public health. and it is essential that the agency understand the application of new technologies to the food supply. The public has become increasingly aware of the advantages of a healthy diet. and. at the same time, increasingly suspect of new development. New foods will not be accepted in the market unless consumers perceive that the products are safe.

\section{What can the agricultural community do?}

What can the agricultural community do to enhance our food supply and to ensure that new developments that are safe are accepted by the public? First expanding and improving our food supply should be a primary goal. An article in The Washington Post (30 Oct. 1989) cited a new report by the National Research Council, "The Lost Crops of the Incas," which mentioned more than 2 dozen native Andean crops still in use today in remote regions that could possibly be adapted in U.S. agriculture to expand our food supply. Some of the crops cited, such as the cherimoya, also known as the "pearl of the Andes", are already reaching U.S. markets.

The use of modem molecular techniques to produce new culti vars offers tremendous potential to improve the nutrition and safety of our food. While the first examples of new biotechnology will be largely aimed at developing improved agronomic traits, such as disease resistance and herbicide tolerance, future improvements in crops to reduce native toxicants could have a substantial impact on the safety of food. Robert Scheuplein. of FDA's Center for Food Safety and Applied Nutrition has examined risks to cancer from a number of causes including traditional food, pesticides and contam inants, mycotoxins. and others. One of his conclusions was that carcinogens in the diet are derived primarily from conventional food, rather that from pesticides. food additives, or contaminants. as is often thought. This being the case. he concludes that reducing the risk from natural carcinogens in food only a few percent could promise "a greater decrease in absolute cancer risk that the total elimination of the lesser risks" (Scheulpein. 1990). The application of new molecular techniques to modify pathways that lead to native carcinogens may play an important role in improving the safety of food. 
Second. agricultural breeding has a history of safety, and this has led to considerable confidence by the public in new cultivars.

"Plant a radish; get a radish.

Never any doubt.

That's why I love vegetables.

You know what you're about." (Jones and Schmidt, 1964)

Such confidence in plant breeding was contrasted to the unpredictable results of breeding children in be song "Plant a Radish" Jones and Schmidt, 1964).

In spite of this confidence. we must find better ways to assess the safety of food because the issues can be difficult to identify precisely and to address scientifically. The key to effective safety evaluation will be to identify and answer the relevant questions in each case. However. one cannot expect to apply the same level of examination to each new cultivar. A recent report by the National Research Council indicates that a plant breeder of corn, soybean, wheat. or potato may introduce as many as 50,000 new cultivars each year (National Research Council, 1989). Therefore, some means will be needed to identify and categorize those new applications that require specific review and to identify the scientific issues that should be addressed Several efforts have been made to accom plish this. The United Kingdom's Ministry of Agriculture, Fisheries, and Food has published guidelines for testing novel foods (U.K. Advisory Committee on Irradiated and Novel Foods) that provide general considerations for chemical composition and nutri tional and toxicological studies in animals and humans. Recently, the International Food Biotechnology Council (IFBC) circulated a draft document (IFBC, 1989) on the regulation and safety assessment of food products derived by biotechnology to various government agencies. public groups, and academia The IFBC is an association of large food professing firms and small biotechnology com panies whose goal is to develop a set of scientific criteria to evaluate the safety of food and food ingredients (plants and microorganisms) developed through applications of biotechnology. The draft report proposes schemes for assessing the safety of both microorganisms and whole foods. FDA appreciates the complexity of the task that the IFBC has undertaken. and believes that the draft report deserves careful and rigorous review.

FDA encourages those developing new food products to consult with agency scientists-early and often-concerning questions that may bear on the safety or regulatory status of the food. It is possi ble that some new foods or food ingredients will not comply with FDA regulations or there may be scientific questions that should be resolved. Because the safety of food can be affected by many factors, and because it is industry's responsibility to establish the safety of food products, it is very important for those companies to work with the agency to ensure that their products are safe and comply with the law.

Third, risk communication will be an important force affecting new developments in agriculture and the food industry in the next few years. Effective risk communication provides the consumer with the tools to evaluate risks in the food supply, to recognize and distinguish risks they are willing to tolerate, and make reasoned choices. Scientists use one set of criteria for judging risk in the food supply; consumers apparently use quite another. Clearly, the two groups are using different criteria to make the same determination of what is safe. Which is right? Neither-there is no right or wrong-the only wrong is failure to communicate.

Consumers expect their food to be free of potentially toxic compounds. However. no food can be guaranteed safe. Consumers need reliable information about risk so they can put it into perspec- tive. Research plays an important role in allaying consumer concerns. Public perception plays a huge role in deciding what the Congress may legislate and regulatory agencies may have to imple ment It is incumbent on food professionals to develop effective methods of risk communication in the coming years.

Although the challenge of biotechnology is tremendous, we do face the challenge of instilling public confidence. As FDA Commissioner Frank E. Young has stated, we need a broadly educated. knowledgeable public capable of making informed decisions of important scientific issues. We must educate the public to understand and appreciate-not fear-the great opportunities that science promises.

\section{Concluding remarks}

In closing, the next several decades will represent a dramatic change in the way we grow, process, and distribute food We must change in the way we grow, process. and distribute food. We must all work together to improve our understanding of and expertise in sciences basic to food safety. The complexity of the food supply demands collaboration to focus the full complement of scientific expertise on the issues-that is, the unique capabilities of each entity joined in cooperative efforts to ensure the safety and quality of the food supply.

As FDA prepares for the future, the agency will determine how it will deal with new technologies that permit new products similar, but not identical to, traditional products; how it will improve its risk process and deal with existing food safety laws; and how it will develop effective risk communication strategies. Technological advances in the last few years have shown FDA that the agency must balance protecting and informing the public with encouraging innovation.

In the likely future continuation of budget constraints, not only in the federal government but in industry and academia as well, it is vitally important that critical issues be identified and that resources be directed in those areas where they will reap the greatest benefits. The more knowledge and data available to FDA for evaluating problem situations, the more rational and scientific will be the solutions. If the answers to pressing questions can not be found, the consequences will almost certainly be increasing conservatism on the part of those of us required to make decisions in these areas.

\section{Literature Cited}

Federal Register. 1970. Fed. Reg. 35:18623.

Federal Register. 1986. Coordinated framework for regulation of biotechnology. Fed. Reg. 51:23302.

IFBC. 1989. Biotechnologies and food: Assuring the safety of foods produced by genetic modification: Draft. International Food Biotechnology Council, Washington. D.C.

Jones, T. and H. Schmidt 1964. The Fantastiks 95. as cited in: Brace. M.J. 1984. Regulation of genetically engineered foods under the federal Food, Drug. and Cosmetic AU. Amer. Univ. Law Rev. 33(4):899.

National Research Council. 1989. Field testing genetically modified organisms: Framework for decisions. National Academy Press, Washington. D.C

Reitz. LP. and B.E. Caldwell 1974. Breeding for safety in field crops, in the effect of FDA regulations (GRAS) on plant breeding and processing Crop Sci. Soc. Amer. Spec. Publ. 5.

Scheuplein. RJ. 1990. Perspectives on toxicological risk: An example: Food-borne carcinogenic risk. (In press.) 